\title{
Particle size dynamics in abrading pebble populations
}

\author{
András A. Sipos ${ }^{1,2}$, Gábor Domokos ${ }^{1,2}$, and János Török ${ }^{1,3}$ \\ ${ }^{1}$ MTA-BME Morphodynamics Research Group Budapest University of Technology and Economics, Múegyetem rakpart 1-3, \\ Budapest, Hungary \\ ${ }^{2}$ Department of Materials and Structures, Budapest University of Technology and Economics, Múegyetem rakpart 1-3, \\ Budapest, Hungary \\ ${ }^{3}$ Department of Theoretical Physics, Budapest University of Technology and Economics, Budafoki út 8, Budapest, Hungary \\ Correspondence: András A. Sipos (siposa@eik.bme.hu)
}

\begin{abstract}
Abrasion of sedimentary particles in fluvial and aeolian environments is widely associated with collisions encountered by the particle. Although the physics of abrasion is complex, purely geometric models recover the course of mass and shape evolution of individual particles in low and middle energy environments (in the absence of fragmentation) remarkably well. In this paper, utilizing results of this individual, geometric abrasion theory as a collision kernel, following techniques

5 adopted in the statistical theory of coagulation and fragmentation, we construct the corresponding Fokker-Planck equation as the first model for the collision-driven collective mass evolution of sedimentary particles. Our model uncovers a startling fundamental feature of collective particle size dynamics: collisional abrasion may, depending on the energy level, either focus size distributions, thus enhancing the effects of size selective transport or it may act in the opposite direction by dispersing the distribution. This complex behaviour fits geological observations on mass distributions.
\end{abstract}




\section{Introduction}

\subsection{Geological observations}

Probably the most fundamental observation on pebbles is that they appear to be segregated both by size and shape and it is broadly accepted that the dynamics is driven by two physical processes: transport and abrasion. Which of these processes dominates may depend on the geological location and also on timescales, however, geologists appear to agree that, in general, neither process should be ignored.

One of the most remarkable accounts of pebble size and shape distribution is provided by Carr (Carr, 1969) based on the measurement of approximately a hundred thousand pebbles on Chesil Beach, Dorset, England. In summarizing his results, Carr provides mean values and sample variations for maximal pebble size and pebble axis ratios along lines orthogonal to the beach. These plots reveal pronounced segregation by maximal size and shape, i.e. on shingle beaches pebbles of roughly similar maximal sizes and with roughly similar axis ratios appear to be spatially close to each other. Size and shape segregation has been broadly observed in various settings (Bird, 1996; Gleason et al., 1975; Hansom and Moore, 1981; Kuenen and Migliorini, 1950; Neate, 1967) and it was mostly attributed to the global transport of pebbles by waves (Lewis, 1931; Carr, 1969) but, in some settings, may also be related to abrasion. Indeed, a detailed account of the interaction of abrasion and transport is given by Landon (Landon, 1930) who investigated the beaches on the west shore of Lake Michigan. He attributes size and shape variation to a mixture of abrasion and transport. Kuenen (Kuenen, 1964) discusses Landon's observations, however disagrees with the conclusions and attributes size and shape variation primarily to transport. Carr (Carr, 1969) observes dominant sizes and shape ratios emerging as a result of abrasion and size grading while Bluck (Bluck, 1967) describes beaches in South Wales where equilibrium distributions of size and shape are reached primarily by transport and abrasion plays a minor role. Which of the two processes (transport or abrasion) dominate may well depend on the timescales they operate on. While abrasion appears in some scenarios to act much slower than transport, a recent study (Bertoni et al., 2016) verified mass losses on the order of $50 \%$ on a pebble beach over a 13-months period, indicating that in some settings the two processes may indeed compete in determining size and shape distributions.

In addition to observing sharp peaks in size and shape distributions, there is yet another common geomorphological scenario to the understanding of which collective abrasion theory may contribute. The often observed presence of isolated large boulders in rivers may be explained solely by transport, as these large pieces are often not carried by the river, rather, they move by a different process (e.g. landslide or debris flow). On the other hand, these large rocks could also be interpreted as outliers emerging spontaneously in a pebble size distribution on which collisional abrasion certainly has strong impact in upper reaches of rivers.

As we can see, a complex picture emerges from geological observations which suggest that the interaction of transport and abrasion may be far from trivial, raising the need to consider both processes (transport and abrasion) simultaneously when trying to explain geological observations on sedimentary particles. 


\subsection{Existing theory and notation}

\subsubsection{Individual abrasion}

Individual abrasion is a theory describing the mass and shape evolution of one individual particle (abraded particle) under the impacts of many incoming particles (abraders) (see Figure 1(a)). In the mean field theory for the geometry of individual abrasion only the mass and shape of the abraded particle is recorded, the effect of impacts is averaged and the evolution is determined by the size of the abraded particle compared to the average size of the abrading particles.

The mean field geometric theory of individual abrasion (i.e. shape evolution) for sedimentary particles under collisions is, since the seminal papers by Firey (Firey, 1974) and Bloore (Bloore, 1977), well understood and validated (Szabó et al., 2013; Szabó et al., 2015; Novák-Szabó et al., 2018). Still, despite the success of the Firey-Bloore geometric theory of shape evolution it was clear (Domokos and Gibbons, 2013) that it is not suited to predict the evolution of size: in stark contrast with geological observations summarized in Sternberg's Law (Sternberg, 1875), predicting exponential decay of particle mass and infinite lifetime for all particles, geometric abrasion theory predicted finite lifetime for all particles. On the other hand, Sternberg's broadly accepted theory of mass evolution (Sternberg, 1875) had nothing to offer regarding the evolution of shape. Recognizing this challenge, in (Domokos and Gibbons, 2013) a unified theory, called volume weighted shape evolution has been proposed which, on one hand, reproduces all the geometric features of the Firey-Bloore geometric theory, on the other hand, it also predicts mass evolution in accordance with Sternberg's Law.

\subsubsection{Binary abrasion}

The stepping stone between the theory of individual abrasion and collective abrasion is the model for mutual, binary abrasion where two particles mutually abrade each other and we track both evolutions (see Figure 1(b)). In this case one can still write mean field equations by averaging over many collisions and the mass and shape evolution of both particles are recorded. For any binary abrasion model of size evolution, we postulate the following requirements:

- size evolution should follow Sternberg's Law,

- mass loss in a collision should be a monotonically increasing function of collision energy and

- the model should be fully compatible with the geometric evolution model.

The unified theory in (Domokos and Gibbons, 2013) offers a model satisfying all three requirements: by extending the FireyBloore equations and Sternberg's theory and using the kinetic energy of collision, models for binary shape evolution and for binary mass evolution of two mutually abrading particles were put forward. These two models have been merged in (Domokos and Gibbons, 2013) into a unified volume weighted theory of binary abrasion, compatible both with the Firey-Bloore and with the Sternberg theory. The volume weighted model for binary mass evolution, describing the time evolutions $X(t), Y(t)$ for the 
(a)

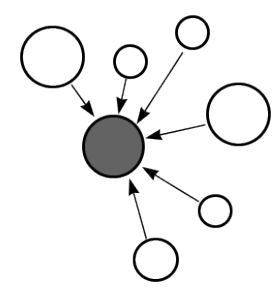

(b)

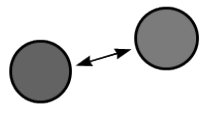

(c)

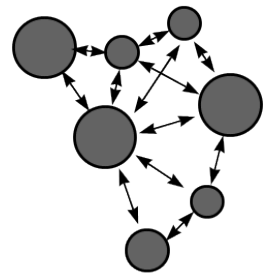

Figure 1. Schemes for (a) individual abrasion, (b) binary abrasion and (c) collective abrasion, respectively. Volume loss only tracked for shaded particles. Arrows represent (non-simultaneous) collision events between particles.

two particle masses can be written as

$$
\begin{aligned}
X_{t} & =-c_{1} \frac{X^{1+r} Y^{1-r}}{X+Y}, \\
Y_{t} & =-c_{2} \frac{Y^{1+r} X^{1-r}}{X+Y}
\end{aligned}
$$

where subscript refers to differentiation and $r$ is a scalar parameter, the role of which we will discuss in detail in Subsection 2.4 .

5 This model uses the hypothesis that on the average, relative collision speed does not depend on mass. The prefactors $c_{i}$ depend on the material and dynamical parameters. If we assume identical material properties for both pebbles then we have $c=c_{1}=c_{2}$ and the sole role of the constant $c$ is to set the timescales. We will incorporate this into the time variable $t$ and henceforth we set $c \equiv 1$.

\subsubsection{Collective size dynamics}

Independently of individual (and binary) abrasion theory there exists broad interest in collective shape and size evolution models tracking mutually colliding populations of $N$ particles (see Figure 1(c)). Similar problems arise in particular in the context of coagulation (da Costa, 2015) and dynamic fragmentation processes (Cheng and Redner, 1988). In such collective evolution models the main question is how the size distribution of particles, starting from an initial distribution, evolves in time due to the mutual collisions. These models use a standard framework relying on a so-called collision kernel. In a more general setting, the collision kernel is referred to as the interaction kernel. Our choice of terminology is motivated by the fact that in our case the only interactions are collisions. Strictly speaking, the collision kernel is the physical model of the $N=2$ case where two particles interact, i.e. it is the corresponding binary evolution model. However, we make the assumption that for large $N$ the collective size evolution is a stochastic process driven by many binary events among the particles, implying that the core of the collective process is still the above mentioned collision kernel. This allows for the construction of the master equation, also known as the Fokker-Planck equation which describes the time evolution of the particle size distribution. Although the collective abrasion is a stochastic process, in the $N \rightarrow \infty$ limit the collision kernel will uniquely determine the global evolution 
of the continuous size distribution. The master equation (or Fokker-Planck equation) is expressing this evolution. Determining the master equation based on the collision kernel is the second step in the statistical model.

The above-outlined structure is characteristic for coagulation fragmentation models (da Costa, 2015), in particular for nonlinear fragmentation, which describe fragmentation processes triggered by binary collisions of particles. Our model may be regarded as a special case of the non-linear fragmentation models (Cheng and Redner, 1988) since, in addition to the standard framework adopted in these models we also make two simplifying assumptions:

1. we only consider collisions where the relative mass loss is small (i.e. the particles lose only fragments with small relative mass) and

2. the small fragments generated in the collisions are not considered further in the evolution.

By implementing these two assumptions into the statistical model based on the collision kernel (1), we take the first step towards establishing the statistical theory of collective size and shape evolution of sedimentary particles. This approach offers multiple methodological advantages. On one hand, by using (1) as the collision kernel, our statistical model will be compatible with Sternberg's Law so we can expect the collective evolution also to observe this theory, albeit in a statistical sense. On the other hand, we can also expect all our results to be compatible with an extended (future) theory which also describes collective shape evolution based on the unified, volume weighted geometric theory in (Domokos and Gibbons, 2013).

\subsubsection{Basic notations}

To describe our construction we will need to address both the size evolution of individual particles (under the collision kernel) as well as the evolution of size distributions. While particle size appears in both settings, we need to distinguish carefully: in individual and binary models particle size evolves in time, in collective models size distribution evolves in time. As a consequence, in the individual setting the variable denoting size may be differentiated with respect of time, in the collective setting this is not the case. We will use $X, Y$ to denote individual particle sizes (either volume or mass) and we will use $x, y$ to denote the independent variables of size distributions. Time evolution of individual particle size will be denoted by $X(t), Y(t)$ with time derivatives $X_{t}(t), Y_{t}(t)$ (subscript will refer to differentiation throughout the paper). The time evolution of size densities will be denoted by $f(x, t), f(y, t)$ with time derivatives $f_{t}(x, t), f_{t}(y, t)$ and size-derivatives $f_{x}(x, t), f_{y}(y, t)$. We denote the expected value and variance of these size distributions respectively by $E(t)$ and $W(t)$ and we will primarily use the relative variance $R(t)=W(t) / E(t)^{2}$ to characterize the evolution of the distributions.

\subsection{Main results}

The collision kernel (1) for mass evolution in (Domokos and Gibbons, 2013) has one single parameter $r$ which is inherited by the corresponding Fokker-Planck equation (shown in subsection 2.2). As we will describe in section 2, the parameter $r$ may, depending on interpretation, represent either the size dependence of the number of collisions or, alternatively, the size dependence of collision energy. Regardless of the interpretation, in subsection 2.3 we find that the that the value $r=0.5$ is critical as it separates two regimes of collective abrasion with qualitatively different evolution $R(t)$ of the relative variance : 


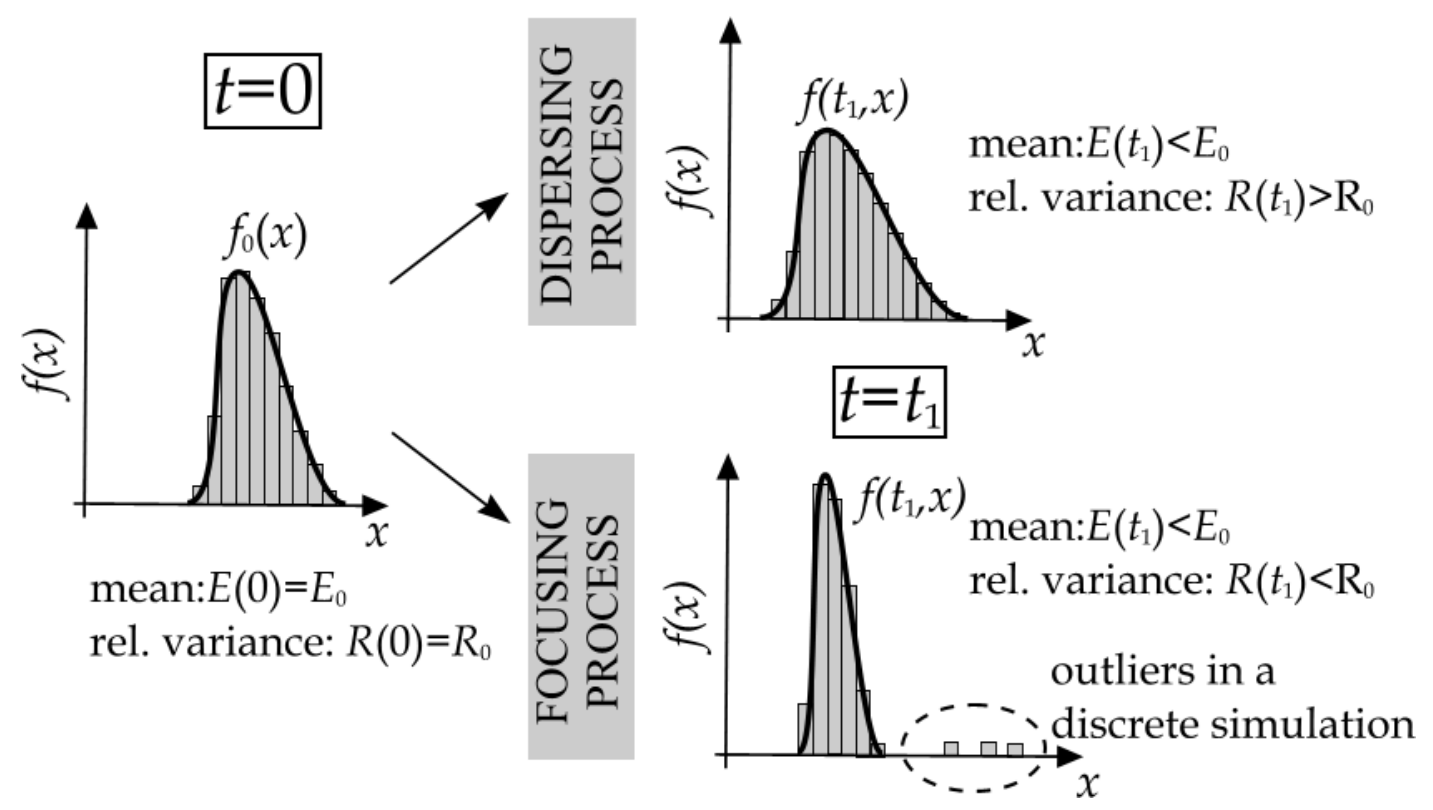

Figure 2. Schematic description of the evolution of mass distribution of a pebble population: in a dispersing process the $R(t)$ relative size variation of the a mass distribution, either represented by an empirical histogram (Carr, 1969), or a continuous function $\left(f_{0}(x)\right)$ at $t=0$, is increasing. In a continuous focusing process $R(t)$ decreases as time evolves, however, in a discrete setting some outliers (indicated by dashed ellipse) with mass substantially above the average. The reduced distribution (without the outliers) produces a decreasing relative variance, analogous to the continuous model.

- For $r>0.5$ we find focusing processes with decreasing $R(t)$, approaching $R(t)=0$ in the limit as time approaches infinity. Here the size distribution converges to a Dirac delta function. This parameter range corresponds to lower energy levels. Natural abrasion processes belonging to this regime will thus amplify the segregating effects of size-selective transport.

- For $r<0.5$ we find dispersing processes with increasing $R(t)$, thus counter-acting size-selective transport processes. This corresponds to collisional abrasion at higher energy levels.

As collisional abrasion may occur at a broad range of energies, these two basic scenarios of the model (illustrated in Figure 2) offer an explanation for the broad range of geological observations (Bluck, 1967; Landon, 1930; Carr, 1969; Kuenen, 1964) in relating the relative significance of transport and abrasion in various scenarios. Our model also reflects the universality of Sternberg's Law by predicting, regardless of the parameter $r$, exponential decay as the universal evolution $E(t)$ of the expected value.

In general, the evolution equations generated by (1) for the mean $E(t)$ and the variance $W(t)$ are integro-differential equations which are hard to solve analytically. To support our claims, we will use three types of approximations: 
(a) We approximate the kernel (7) by its truncated Taylor series expansion and investigate the evolution of general initial density functions. This is found in Appendix A3.

(b) We regard the full kernel, however, we only investigate density function obtained as a small perturbation of the Dirac delta (i.e. populations of almost identical particles). This is done in Appendices A4 and A5.

(c) We numerically compute both the discrete and the continuum models. For details see Section 3.

We will briefly refer to the first two approximations as the continuum model. In case of the third approximation we do direct, discrete simulations of finite particle populations we use the full kernel and we call this the discrete model. One startling feature of the latter (as compared with the former) is the appearance of outliers, i.e. particles substantially larger than the vast majority (illustrated in Figure 2). As we can observe, the bulk of the density function closely mimics the evolution in the continuum model. The quantitative analogy in the evolution of the relative variation $R$ can be also recovered if we consider a reduced density function $f^{\star}\left(t_{1}, x\right)$ by omitting the outliers, i.e by applying an upper cutoff in size, omitting bins containing only one particle. The reduced density function $f^{\star}\left(t_{1}, x\right)$ is characterized by the reduced relative variation $R^{\star}$ which will decrease in a focusing process, however, in contrast to the continuum model, it will not approach zero, rather a positive constant.

\section{Modeling collective size dynamics}

\subsection{General form of the collision kernel}

The first simplification described in subsection 1.2.3 implies that the limit where relative fragment mass approaches zero offers a good approximation, thus it admits a collision kernel of the type used in Ernst and Pagonabarraga (2007), describing continuous mass evolution via coupled ordinary differential equations for the evolution of particles with masses $X(t)$ and $Y(t)$ :

$-X_{t}=\psi^{1}(X, Y)$

$-Y_{t}=\psi^{2}(X, Y)$

where $\psi^{1}(X, Y)$ and $\psi^{2}(X, Y)$ are differentiable $\left(C^{1}\right)$ functions, with positive values (i.e. $\left.\mathbb{R}^{+} \times \mathbb{R}^{+} \rightarrow \mathbb{R}^{+}\right)$. Symmetry of the binary process implies $\psi^{1}(X, Y)=\psi^{2}(Y, X)$, so often superscripts are suppressed and the kernel is simply referred to as $\psi(.,$.$) . Selection of the kernel encapsulates not only the physics of binary collisions, it also may include the mass-dependent$ probability of collision between two particles. We will discuss the identification of a physically sound kernels in subsection 2.3.

\subsection{General form of the master equation}

The second simplification in subsection 1.2.3 admits the construction of the master equation solely based on the collision kernel (by omitting additional terms for the remainder of the fragmented material). These simplifying assumptions also set our model apart from general fragmentation models in another respect: in the latter, constant mass is prescribed as a global time-invariant 
while the (integer) number of particles changes whereas in our model total mass is decreasing while the number of particles remains constant and serves as a global invariant.

Using these considerations, for our problem the master equation is found to be

$f_{t}(t, x)=\frac{\partial}{\partial x}\left[f(t, x) \int_{0}^{\infty} f(t, y) \psi(x, y) d y\right]=f_{x}(t, x) \int_{0}^{\infty} f(t, y) \psi(x, y) d y+f(t, x) \int_{0}^{\infty} f(t, y) \psi_{x}(x, y) d y$

5 where subscripts stand for partial derivatives. Without loss of generality, the evolution starts at $t=0$ and we consider the initial distribution of the volume $f(0, x) \equiv f_{0}(x)$ to be a-priori known. Note that, contrary to the majority of Fokker-Planck models, spontaneous fragmentation is not allowed in our model, eliminating diffusion.

We aim to understand some scenarios characteristic of pebble populations by investigating the Cauchy-type initial value problem associated with equation (4), starting at the distribution $f_{0}$ with mean value $E_{0}$, variance $W_{0}$ and relative variance $R_{0}:=W_{0} / E_{0}^{2}$

\subsection{Collision kernels}

Detailed physical modeling of the collisional event can make the interaction kernel highly complex; for a recent review on kernels see (Meyer and Deglon, 2011). On the other hand, mathematical studies tend to prefer simple expressions for $\psi(X, Y)$, admitting rigorous, analytical conclusions. Our goal is to a find kernel which has strong physical basis, yet it admits an analytical approach, thus it offers a trade-off between between physical and mathematical preferences.

We first consider two simple kernels which satisfy the mathematical requirement of leading to analytically soluble FokkerPlanck equations. However, as we will show, these very analytical results highlight that these kernels are physically not admissible. Next, we investigate the parameter dependent compound kernel suggested in (Domokos and Gibbons, 2013) which grabs the essential physics of the investigated process, yet, the corresponding Fokker-Planck equation still admits analytical conclusions.

First, we consider the summation kernel (denoted by $\{.\}^{+}$), where the mass loss rate is proportional to the sum of the masses of the colliding particles:

$\psi^{+}(X, Y):=X+Y$

stating that the rate of mass loss in binary collisions is proportional to the total mass of the two colliding particles. Appendix

25 A1 demonstrates that the relative variance of the mass in case of the summary kernel follows $R^{+}(t)=R_{0} e^{2 t}$, hence it is a dispersive process regardless of the initial distribution $f_{0}$.

In the very same manner let us investigate the product kernel distinguished by the sign $\{.\}^{*}$. The product kernel is defined via

$\psi^{*}(X, Y):=X Y$

According to Appendix A2, the relative variance in this case is constant as $R^{*}(t)=R_{0}$ for all $t \geq 0$, which that the model is nor focusing neither dispersing. Note that the time-invariance of $R^{*}(t)$ under the product kernel does not imply the invariance 
of the P.D.F $f(t, x)$ per-se. In addition, we see a polynomial decay in the mass as $E^{*}(t)=\left(t+E_{0}^{-1}\right)^{-1}$, which contradicts Sternberg's law (Sternberg, 1875) that postulates an exponential decay.

In order to be in accordance with Sternberg's law and to have a control on the evolution of the relative variance, following the lead of (Domokos and Gibbons, 2013) we investigate the interaction law (1) which we call a compound kernel and using the introduced general notation for kernels, we distinguish it with the $\{.\}^{c}$ sign:

$\psi^{c}(X, Y):=\frac{X^{1+r} Y^{1-r}}{X+Y}$,

where $0 \leq r \leq 1$ is a fixed parameter. Henceforth we investigate evolution of mass density functions under the Fokker-Planck equation derived from (7). The essential properties of the three investigated kernels are summarized in Figure 3.

\subsection{Interpretation of the parameter $r$}

In natural events, both velocity and collision probability (cross- section) may depend on particle size: in laminar flows relative velocity and collision probability is proportional to linear size, while in a turbulent flows velocity could be inversely proportional to linear size and collision probability could be proportional projected area. In the collision kernel (7) both effects (dependence of velocity and dependence of collision probability on speed) are represented by the single scalar parameter $r$, so one may freely assign various interpretations to this parameter. In Domokos and Gibbons (2013) one particular interpretation was used: the compound kernel was derived using the assumption that particle velocity is independent of the size (e.g. rather determined by the surrounding fluid), but the collision probability goes as a power law with particle size, i.e. $X^{r}$. The effective mass combined with the collision probability gives the kernel in Eq. (7). However, alternative interpretations are possible, the only essential underlying assumption is that we regard a one-parameter family of scenarios. In this family, if velocity is proportional to $X^{a}$ and collision probability is proportional to $X^{b}$ then we have $r \simeq a+b$.

To have a global view, it may be of interest to estimate the parameter $r$ in two extreme (limiting) scenarios. Laminar flows are characterized by a linear velocity profile. The particles hit each other if their trajectories intersect. Integration of the linear velocity profile combined with a spherical particle shape yields a collision probability proportional to $r \simeq 2 / 3$. The other extreme case corresponds to turbulent flows, where we have equipartition, i.e. the kinetic energy of the particles is independent of their size (see e.g. Uberoi (1957)), implying that particle velocity is proportional to $X^{-1 / 2}$. Since the area of the cross-section is proportional to $X^{2 / 3}$ we arrive at a collision probability $X^{1 / 6}$, or, alternatively $r \simeq 1 / 6$. As we can see, both extreme scenarios yield $r$ values far away to either side of the critical value $r_{\text {crit }}=1 / 2$, so these estimates suggest that smooth steady conditions should result in a focusing and turbulent gas-like behavior in a dispersing process.

In order to examine the validity of these assumptions we made discrete element simulations using the event driven method Lubachevsky (1991). In event driven dynamics, collisions are considered instantaneous and resolved accordingly which is best suited to obtain proper collision statistics. We emulated the above-mentioned processes by choosing an artificial mass for the particles and simulating a chaotic system. The artificial mass was used to obtain different volume-velocity relations in different scenarios. We found that in chaotic/turbulent systems that relative velocities were proportional to $v \sim V^{-1 / 2}-V^{-1 / 3}$ and the system behaved as the continuum model with $r \sim 1 / 6-1 / 3$. On the other hand, if velocities were proportional to 
a1)

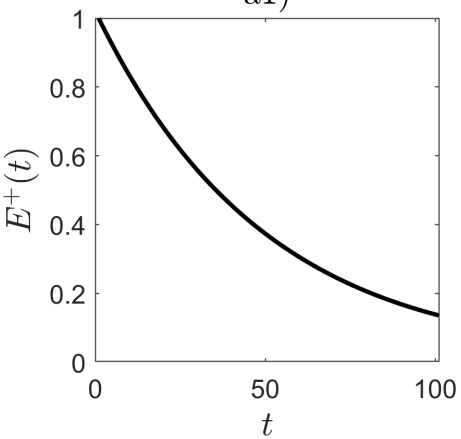

b1)

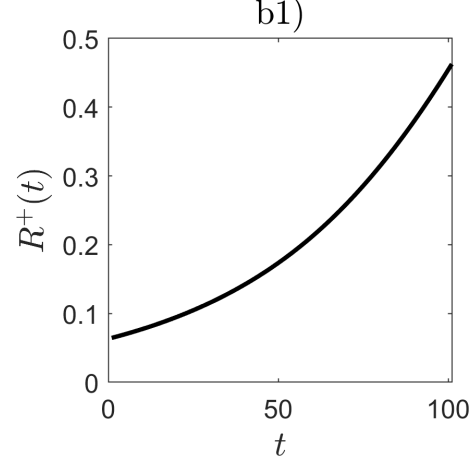

c1)

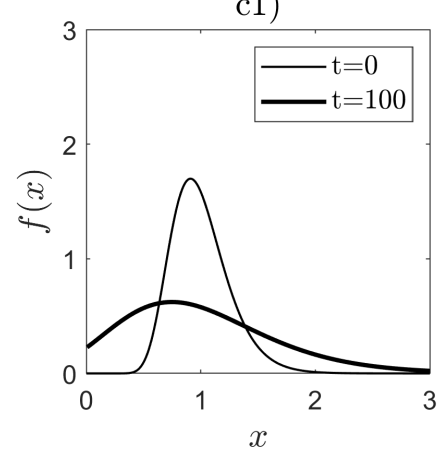

a2)

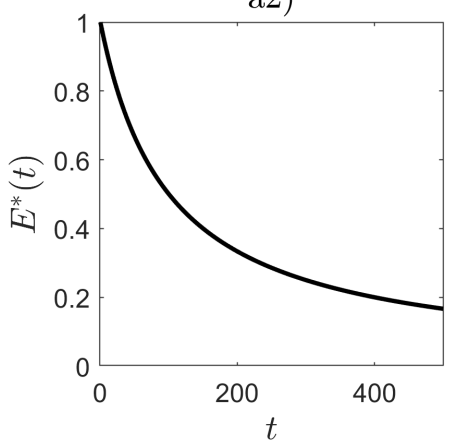

b2)

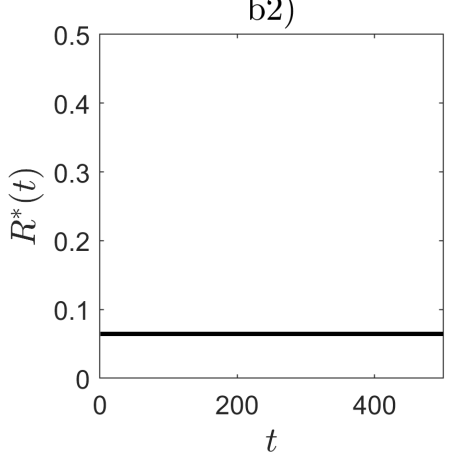

c2)

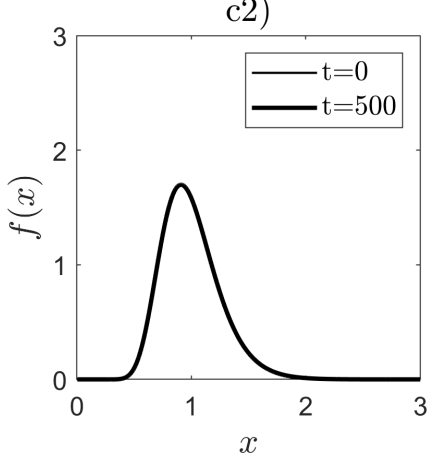

a3)

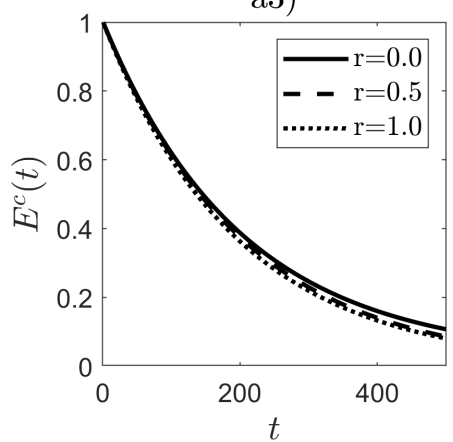

b3)

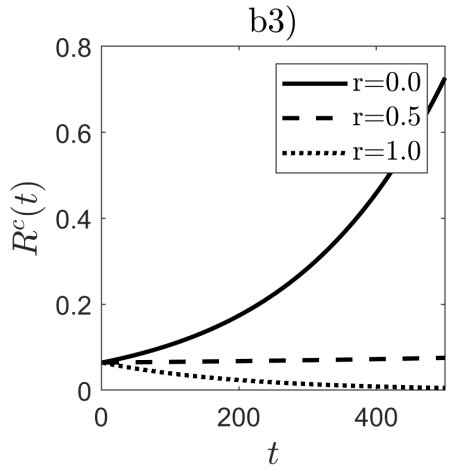

c3)

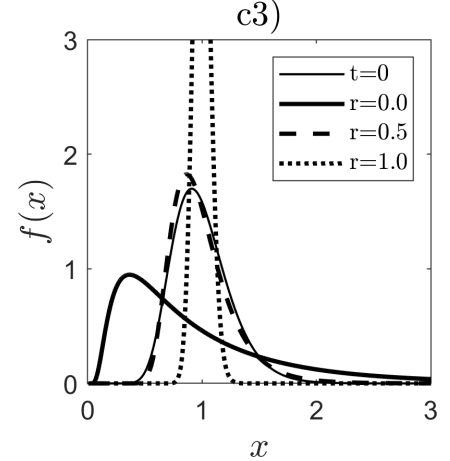

Figure 3. Evolution of an initial $(t=0)$ lognormal probability density function under Fokker-Planck equation generated by various kernels. Summation kernel: a1,b1,c1. Product kernel: a2,b2,c2. Compound kernel:a3,b3,c3. First row (a1,a2,a3): mean $E(t)$. Second row (b1,b2,b3): relative variance $R(t)$. Third row (c1,c2,c3): initial $(t=0)$ and final densities $f(x, t)$.

$v \sim V^{-1 / 6}-V^{0}$ then the system was similar to a continuum model with $r=1 / 2-2 / 3$. Thus the discrete element simulations fully support the results of the compound kernel. 


\section{Numerical results}

Here we perform computations to illustrate the main results presented in subsection 1.3 by discretizing time with a fixed time-step $\Delta t$. The discrete model has been simulated with custom-made codes in Matlab and Python performing $M *[N / 2]$ collisions between pairs during one time-step $\Delta t$, where $M$ is fixed model parameter and $N$ is the size of the population. The simulation starts with the creation of $N$ particles whose volumes are randomly sampled from the initial distribution $f_{0}$. Binary collisions are performed on uniformly selected pairs, i.e., all particles have equal chance of being selected irrespective of their volume. Once a pair is selected, the collision kernel $\psi^{c}$ is applied and volume decrement is computed with time-step $\Delta t / M$. After the binary collision event both particles with reduced volume are replaced into the sample. In the presented simulations we set the population size to be $N=5000$, the time-step $\Delta t=0.01$ and $M=10$. In the continuum setting $f(t, x)$ evolves under eq. (4) with some initial value $f_{0}(x)$. This code uses the operator exponential syntax of a the Chebfun toolbox (Driscoll et al., 2014) in Matlab. The applied time step is fixed at $\Delta t=0.01$.

\subsection{Focusing and dispersing regimes}

The evolution of a pebble population under the compound kernel was simulated both in the frame of discrete and the continuum model, i.e. by direct event-base simulation and by discretizing the partial differential equation. (see Sec. 1.3). The results show excellent agreement with our analytical predictions: $r=1 / 2$ appears indeed a critical parameter in the model. This is illustrated in Figure 4 where a lognormal distribution is used as an initial value for the evolution.
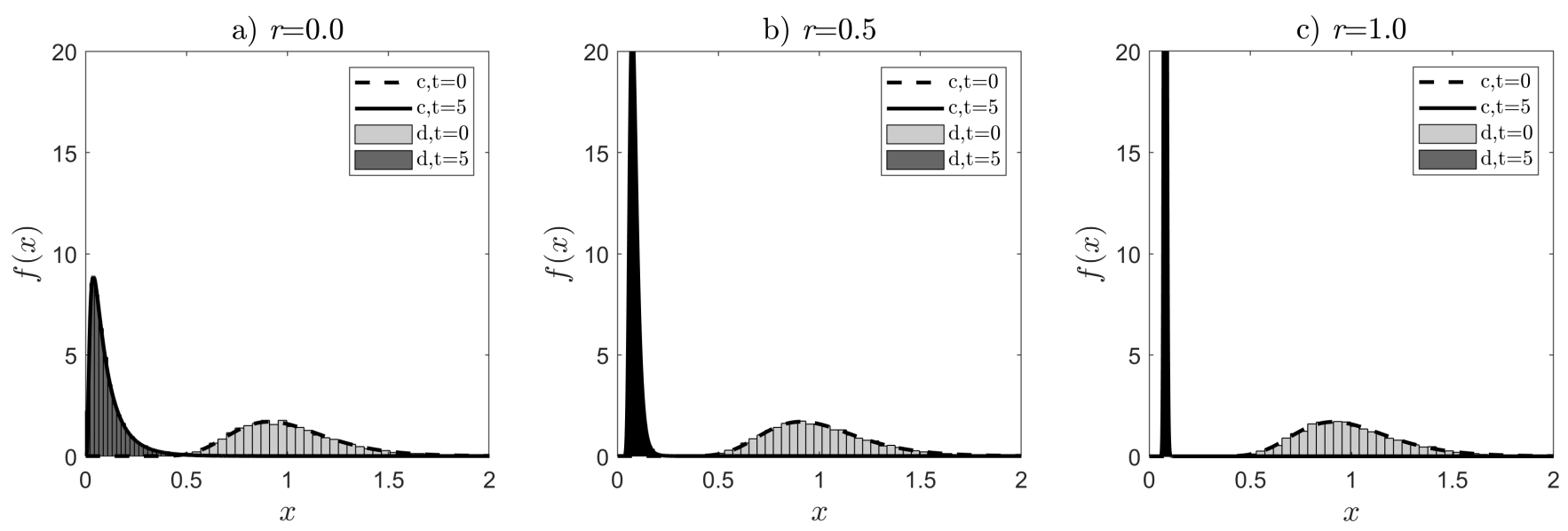

Figure 4. Evolution of a lognormal P.D.F in the compound model, at the parameter values $r=0.0$ (left panel), $r=0.5$ (middle panel) and $r=1.0$ (right panel) from $t=0$ until $t=5.0$. The results of the discrete simulations are given by the histograms, the output of the continuous model are given by dashed (initial distribution) and solid lines (final distribution). Observe the fair agreement between the discrete and the continuous models. 


\subsection{Fitted lognormal distribution}

Although the lognormal distribution is certainly not invariant under the compound kernel (i.e. an initially lognormal density function does not remain lognormal in the evolution), however, mass distributions in later timesteps highly resemble lognormal dsitributions. To test this visual observation we fitted lognormal distributions to the computed mass distributions in the discrete simulations. The evolution of the two parameters (respectively denoted to $\mu$ and $\sigma$ ) of the lognormal distribution are given in Figure 5 at values of the parameter $r$. The criticality of $r=0.5$ is obvious in this setting, too: while the initially lognormal distribution is almost invariant under the evolution at $r=1 / 2$, the evolution of the parameters $\mu$ and $\sigma$ take an opposite direction in the parameter space for $r=0.0$ and $r=1.0$, respectively. The $95 \%$ confidence levels of the fit confirm the visual intuition: the evolved distributions are close to lognormal: in practical applications an approximation with a lognormal distribution produces an acceptable error.
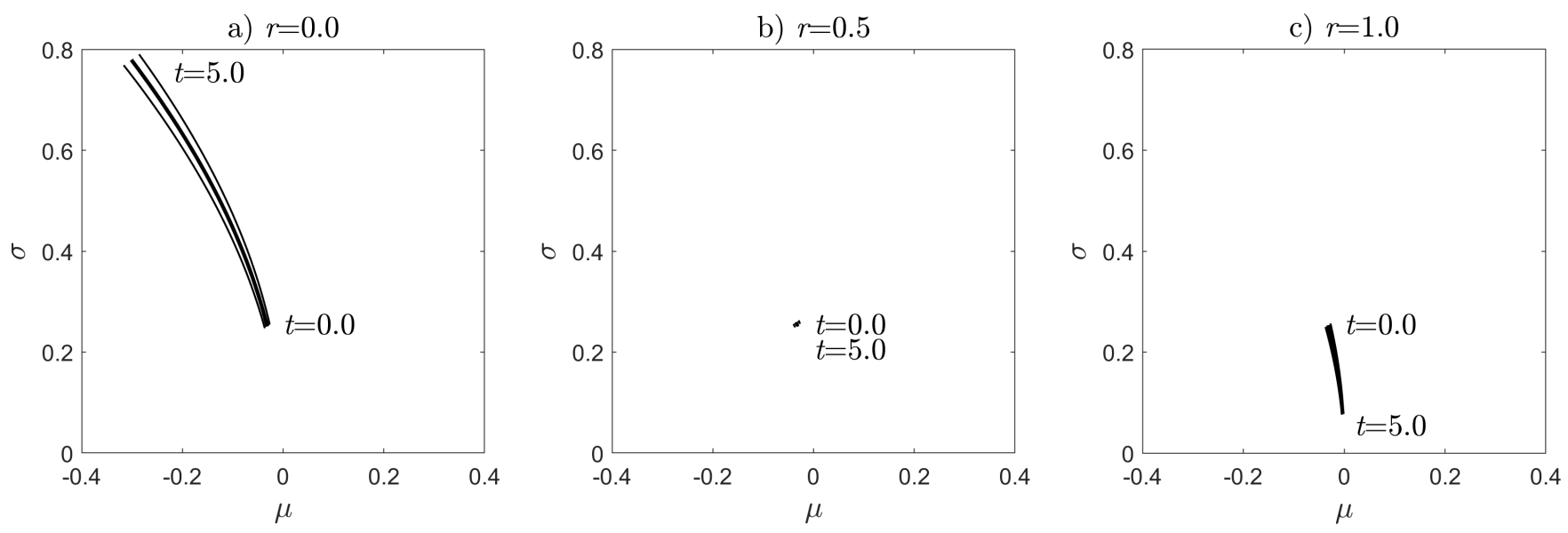

Figure 5. Parameters, $\mu$ and $\sigma$ of a lognormal distributions fitted to the computed mass distribution in the compound model, at the parameter values $r=0.0$ (left panel), $r=0.5$ (middle panel) and $r=1.0$ (right panel) from $t=0$ until $t=5.0$. Thick solid lines correspond to the best fit, thin lines indicate the $95 \%$ confidence level of the fit. Observe the narrow zone spanned by the confidence intervals.

\subsection{Outliers: anomalies in smaller samples}

The continuum model describes the $N \rightarrow \infty$ limit of the system. In the computations shown in subsections 3.1 and 3.2 we either showed results based on the contiuum model or, in the direct, discrete simulations we treated large $(N=5000)$ populations. However, if we look at the discrete simulations on smaller samples we may observe unexpected phenomena not recorded in the previous computations. In Fig. 6 we show the mass distribution of a system at $r=0.6$ with $N=2000$ particles. The bulk of the histograms can be well approximated with a log-normal distribution. However, there are 12 particles with somewhat larger volume than predicted by the lognormal distribution and one approximately 150 times the median volume (5.3 times the radius). Thus inside the focusing regime we may observe a situation where we have a well-defined narrow distribution which 
(b)
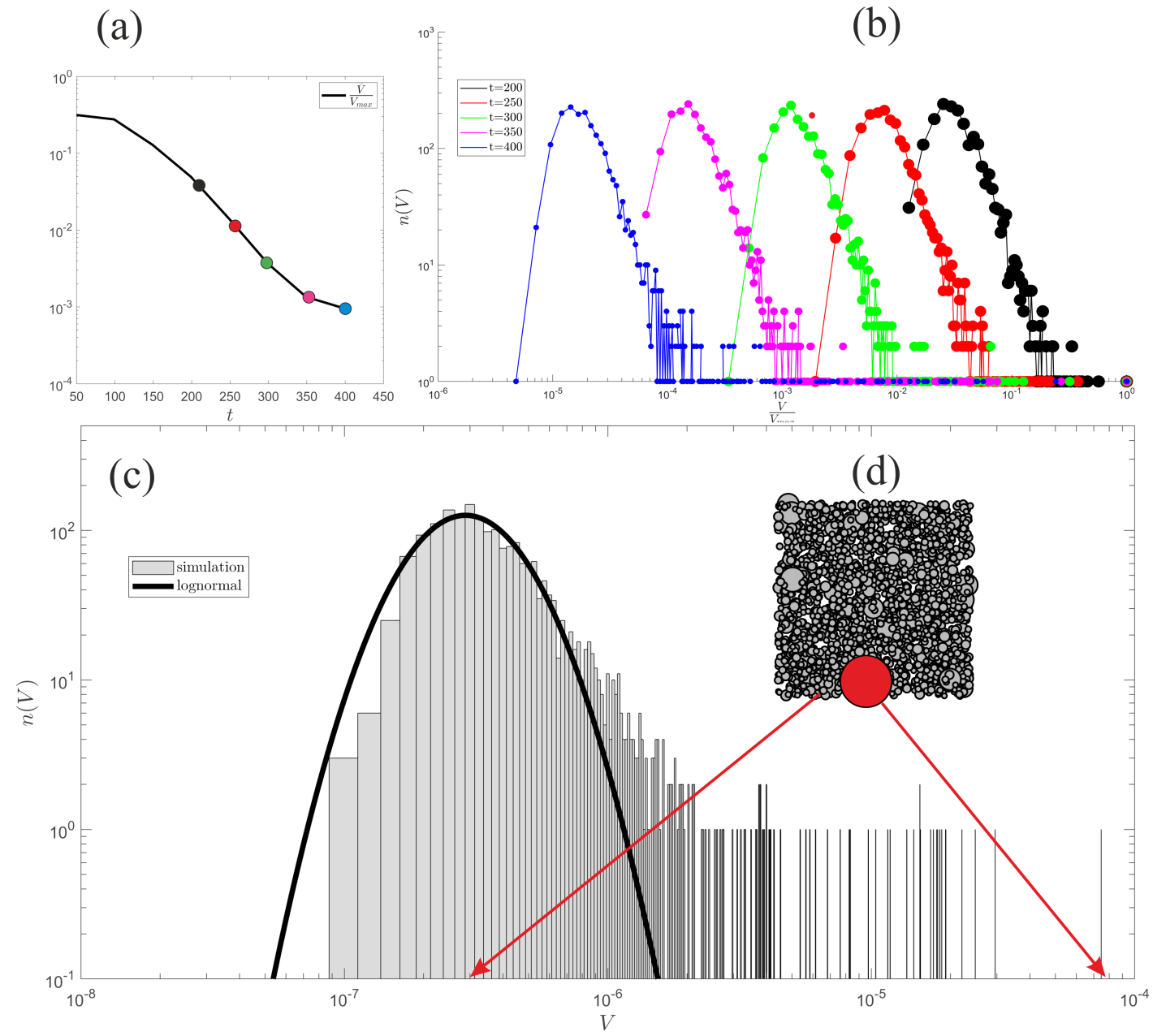

Figure 6. Simulation of a finite sample with $N=2000$ particles. Inset (a) shows the evolution of the mean volume normalized by the maximal volume. Inset (b) depicts the evolution of the distribution, the corresponding points in (a) are denoted by the same color.The green curve $(t=300)$ is the one shown in detail in panel (c), it depicts the particle volume histogram after 300 collisions per particle. The grey boxes show the logarithmically binned histogram, the black line is a log-normal fit to the data. Observe the existence of outliers on the right. Inset (d) is a visual illustration of the entire population: all particles are placed randomly into a $2 \mathrm{~d}$ container. Smaller particles were placed first and the white content (gray scale) is proportional to the linear size of the particle. One small particle close to the mean and one large particle (outlier) are marked with red and their position is indicated in the distribution.

describes the bulk of the particles but a few might escape from this process and may be left behind, at larger mass. This effect is persistent and it was observed also for the parameter value of $r \simeq 0.7$.

In order to estimate the robustness of this scenario we use a simple approximation by assuming that all but one particles have volume $X$ and one single, exceptional particle, called 'outlier' has a volume $a X$ with $a \gg 1$. As it is demonstrated in Appendix 
A6, the outlier can coexist with the population of the small particles. In the $N \rightarrow \infty$ limit the condition of such a coexistence reads

$\frac{2 a^{r}}{1+a} \leq 1$

Numerical solution of (8) for equality yields the critical curve $a_{c}(r)$ on the $[r, a]$ parameter plane, separating systems where outliers may coexist with the population from systems where they may not. While we computed the $a_{c}(r)$ critical curve for the case of infinitely large populations (the $N \rightarrow \infty$ limit), we stress the fact that the illustrated phenomenon is inherently discrete and does not arise in the continuum model. We may explain this curious phenomenon in the following manner: Assume that we start from a narrow distribution. Then random fluctuations in the discrete system may create particles with large relative mass (i.e. large parameter value $a$ ). If these fluctuations are sufficiently large to create particles above the critical curve $a_{c}(r)$ then these outliers will be sustained, otherwise their mass will again approach the average mass of the majority. The critical curve in Figure 7 shows that in the vicinity of the critical value $r=0.5$ almost any such fluctuation will be sustained and outliers are likely to survive. However, as the parameter $r$ is increasing, it gets increasingly less likely to see sustained outliers. Another observation is that as the likelihood for the existence of outliers decreases, their expected relative size is increasing which matches the common-sense observation that the larger the outlier, the less frequently it may be observed.

\section{Conclusions}

In this paper we presented the first statistical model for the collective mass evolution of pebble populations under collisional abrasion. While our model is certainly not unique, it is compatible with

(a) existing geological observations,

(b) existing geometrical theory of individual and binary abrasion of pebbles,

(c) existing theory for individual mass evolution of pebbles (Sternberg's Law) and

(d) exiting statistical theory of coagulation and fragmentation.

In the spirit of standard statistical theory for collective evolution, our model is based on two components: (i) the binary collision kernel and, based on the latter, (ii) the governing equation for the evolution of probability density functions for mass distribution. Regarding (i) we used the model from (Domokos and Gibbons, 2013) which incorporates the existing theory for individual and binary abrasion, regarding (ii) we used the Fokker-Planck equation which is broadly used in the theory of coagulation in fragmentation.

Our collision kernel includes the single scalar parameter $r$ which can be associated with the energy level of the collective collisional evolution process. We found that $r=0.5$ is critical, separating two regimes with fundamentally different behaviour: for $r>0.5$ (low energy regime) we found focusing behaviour with decreasing relative variance $R(t)$ and for $r<0.5$ (high energy regime) we found dispersing behaviour with increasing relative variance $R(t)$. In geological terms, this result suggests 


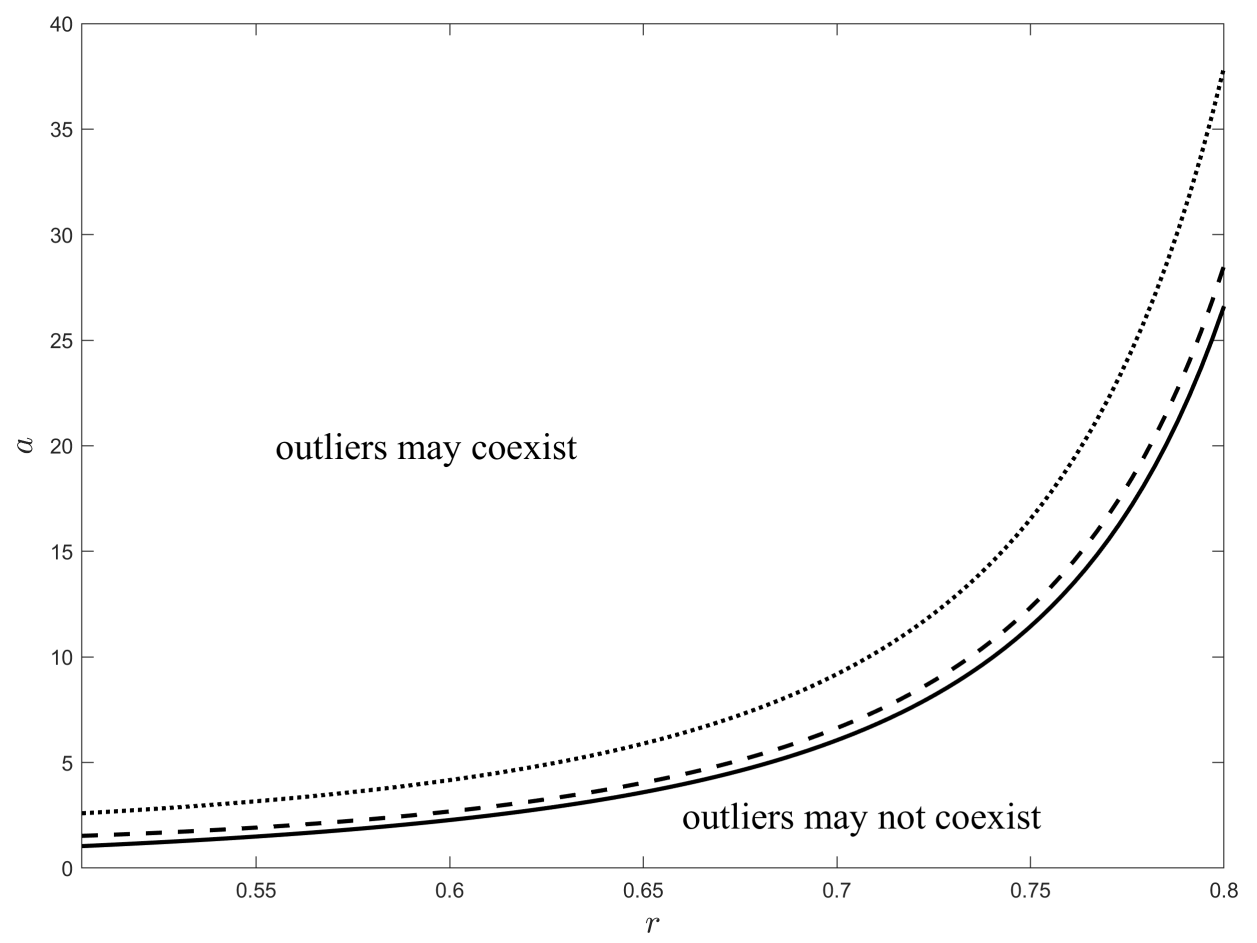

Figure 7. Critical curve $a_{c}(r)$ on the $[r, a]$ parameter plane. Systems with parameters $(r, a)$ associated with points above the curve admits the coexistence of outliers while the systems associated with points below the critical curve do not admit the coexistence of outliers. Solid line belongs to the $N \rightarrow \infty$ limit, dotted line represents $N=20$ and the dashed line $N=100$ particles, respectively.

that in low energy environments collisional abrasion acts on mass distributions in unison with size-selective transport while in high energy environments the opposite happens and the two processes are counter-acting. In accordance with prevailing geological observations and Sternberg's Law,, our models predicts exponential decay of particle mass in both energy regimes.

We investigated our model on two levels: (i) as a continuum model by regarding the evolution of the Fokker-Planck equation 5 and (ii) as a discrete model by running discrete event-based simulations. In case of the continuum model we derived our results analytically and also from numerical simulation of the Fokker-Planck equation while in the discrete model we relied on numerical computations. In regard of the existence of the critical parameter $r=0.5$ and the existence of the focusing and dispersing regimes, the two approaches yielded quantitatively matching results.

Large boulders among many small pebbles are often visible in mountain ranges of rivers. While this phenomenon is commonly attributed to transport, our model suggests that under some conditions, here again transport and abrasion may act in unison: we identified a curios phenomenon not present in the continuum model but present in the discrete model (even in the $N \rightarrow \infty$ limit). If the parameter $r$ was in the focusing $r>0.5$ range but not very far from the critical value $r=0.5$, the bulk of the distribution was narrowing (in accordance with our analytical predictions), however, we could also observe a few particles with substantially larger mass (outliers), escaping the bulk of the distribution. We characterized the mass ratio of outliers versus 
the mean of the bulk distribution by the parameter $a$ and we derived a critical curve $a_{c}(r)$ separating systems where outliers may be observed from those where this may not happen. Our result predicts that larger outliers are less likely to be observable.

While our paper only dealt with on size distributions, however, there exist also related observations on shape: sharp peaks in distributions of axis ratios (also referred to as equilibrium shapes) are mentioned in Bluck (1967); Dobkins and Folk (1970);

5 Landon (1930); Orford (1975); Williams and Caldwell (1988); Ashcroft (1990); Lorang and Komar (1990); Yazawa (1990); Wald (1990). In Domokos and Gibbons (2012) a plausible argument was presented that equilibrium shapes may emerge on shingle beaches as the result of interaction of abrasion and transport. We hope that the extension of the statistical theory presented in this paper may be capable to verify these observations.

\section{Appendix A: Some properties of the kernels in Section 2}

\section{A1 Summary kernel}

Differential equations governing the time evolution of the first and second moments can be readily obtained, hence the mean $E^{+}(t)$ and variance $W^{+}(t)$ follows the following initial value problems (IVPs):

$$
\begin{gathered}
E_{t}^{+}(t)=-2 E^{+}(t) \quad \text { with } \quad E^{+}(0)=E_{0}, \\
W_{t}^{+}(t)=-2 W^{+}(t) \quad \text { with } \quad W^{+}(0)=W_{0} .
\end{gathered}
$$

15 It follows, that both the expectation and the variance exhibit exponential decay, namely $E^{+}(t)=E_{0} e^{-2 t}$ and $W^{+}(t)=$ $W_{0} e^{-2 t}$. It is straightforward to show, that the relative variance $R^{+}(t)$ increases exponentially

$R^{+}(t):=\frac{W^{+}(t)}{E^{+}(t)^{2}}=\frac{W_{0}}{E_{0}^{2}} e^{2 t}=R_{0} e^{2 t}$.

\section{A2 Product kernel}

In case of the product kernel the IVPs describing the evolution of the mean $E^{*}(t)$ and variance $W^{*}(t)$ respectively read

$E_{t}^{*}(t)=-\left(E^{*}(t)\right)^{2} \quad$ with $\quad E^{*}(0)=E_{0}$,

$W_{t}^{*}(t)=-2 W^{*}(t) E^{*}(t) \quad$ with $\quad W^{*}(0)=W_{0}$.

Here the decay of the mean and the variance are polynomial as we find

$E^{*}(t)=\frac{1}{t+\frac{1}{E_{0}}}$, and $W^{*}(t)={\frac{W_{0}}{E_{0}}}^{2} \frac{1}{\left(t+\frac{1}{E_{0}}\right)^{2}}$

which result in a steady relative variance $R^{*}(t)$, determined by the initial distribution $f_{0}$. In specific

$25 R^{*}(t):=\frac{W^{*}(t)}{E^{*}(t)^{2}}=\frac{W_{0}}{E_{0}^{2}}=R_{0}$. 


\section{A3 Truncated compound kernel}

The truncated compound kernel is obtained from the compound kernel as the truncated Taylor polynomial computed at $y=x$ with an $\left(O(y-x)^{2}\right.$ :

$\psi^{c, T}(x, y):=\frac{x}{2}+\left(\frac{1}{4}-\frac{r}{2}\right)(y-x)+O\left((y-x)^{2}\right)$.

5 Using the master equation, the following Cauchy problems are found that define the evolution of the mean and the variance:

$$
\begin{aligned}
E_{t}^{c, T}(t) & =-\frac{1}{2} E^{c, T}(t) \quad \text { with } \quad E^{c, T}(0)=E_{0}, \\
W_{t}^{c, T}(t) & =-\left(\frac{1}{2}-r\right) W^{c, T}(t) \quad \text { with } \quad W^{c, T}(0)=W_{0} .
\end{aligned}
$$

Solution of these ODEs yields the evolution of the relative variance as

$$
R^{c, T}(t):=\frac{W^{c, T}(t)}{E^{c, T}(t)^{2}}=\frac{W_{0}}{E_{0}^{2}} e^{\left(\frac{1}{2}-r\right) t} .
$$

\section{A4 A population of identical particles preserved}

Here we show, that a population of identical particles, characterized by a Dirac delta function as P.D.F. is preserved in the model with the compound kernel regardless of the value of parameter $r$. Without loss of generality, we investigate the evolution from the $f_{0}=\delta(1)$ initial condition, where $\delta(x)$ denotes the Dirac-delta function at $x$. Obviously, $E_{0}=1$ and $W_{0}=0$. We show, that now $f(t, x)=\delta(c(t))$ holds for any $t>0$. Let us assume, that at some $t^{*} \geq 0$ the distribution is $f\left(t^{*}, x\right)=\delta\left(c\left(t^{*}\right)\right)$ with mean $E^{c}\left(t^{*}\right)=c\left(t^{*}\right)$ and variance $W^{c}\left(t^{*}\right)=0$, respectively. Observe that

$\int_{0}^{\infty} f\left(t^{*}, y\right) \psi^{c}(x, y) d y=\psi^{c}\left(x, c\left(t^{*}\right)\right)$.

The time derivative of the mean can be computed via

$E_{t}^{c}\left(t^{*}\right)=\int_{0}^{\infty} f_{t}\left(t^{*}, x\right) x d x=-\int_{0}^{\infty} f\left(t^{*}, x\right) \psi^{c}\left(x, c\left(t^{*}\right)\right) d x=-\frac{1}{2} c\left(t^{*}\right)$

where we used (4), applied integration by parts and employed (A12). Similarly, the evolution of the variance is found to follow:

$W_{t}^{c}\left(t^{*}\right)=\int_{0}^{\infty} f_{t}\left(t^{*}, x\right) x^{2} d x-2 E_{t}^{c}\left(t^{*}\right) E^{c}\left(t^{*}\right)=-2 \int_{0}^{\infty} f\left(t^{*}, x\right) \psi^{c}\left(x, c\left(t^{*}\right)\right) x d x+c\left(t^{*}\right)^{2}=-c\left(t^{*}\right)^{2}+c\left(t^{*}\right)^{2}=0$.

This shows that the variance of the distribution is constant, and as it started at $W_{0}=0$, i.e. it vanishes whole along the evolution. In other words, the we have a Dirac-delta (degenerate) distribution at any $t \geq 0$. Employing (A13) we find, that the location $c(t)$ follows the initial value problem $c_{t}(t)=-\frac{1}{2} c(t)$ with $c(0)=1$, hence $c(t)=\exp \left(-\frac{t}{2}\right)$. 


\section{A5 Dispersing and focusing behaviour identified in the population of almost identical particles}

As the model lacks diffusion, the behaviour of a degenerate distribution with all the mass concentrated at a single value is worthy to study. In Appendix A4 we show that a population of identical particles remain identical in our model. In other words, time-invariance of the Dirac-delta distribution holds in our model, regardless of the value of the parameter $r$. Nevertheless, the value of $r$ affects the stability of that Dirac-delta: next we show that the evolution for a population of almost identical particles (i.e. a perturbed version of the Dirac delta distribution) is either focusing or dispersing, depending on the value of $r$. To see this, we define a perturbed distribution. Let $\varepsilon>0$ be a fixed parameter and define

$\hat{f}_{0}(x):= \begin{cases}(1-\varepsilon) \delta(1)+\frac{1}{2} & \text { if } 1-\varepsilon \leq x \leq 1+\varepsilon \\ 0 & \text { otherwise }\end{cases}$

It is straightforward to show that $\int_{0}^{\infty} \hat{f}_{0}(y) \psi^{c}(x, y) d y=\psi^{c}(x, 1), E_{0}^{c}=1$ and $M_{2}^{c}(t):=\int_{0}^{\infty} f(t, x) x^{2} d x$ with $M_{2}^{c}(0)=1+$

$10 \frac{1}{3} \varepsilon^{3}$. We aim to investigate the sign of $R_{t}^{c}$ at $t=0$. Since $R^{c}(t)=M_{2}^{c}(t) E^{c}(t)^{-2}-1$, we need to study the sign of $M_{2, t}^{c}(0) E^{c}(0)-$ $2 E_{t}^{c}(0) M_{2}^{c}(0)$. Integration by parts yields

$E^{c}(0) M_{2, t}^{c}(0)-2 M_{2}^{c}(0) E_{t}^{c}(0)=-2 \int_{0}^{\infty} \hat{f}_{0}(x) \psi^{c}(x, 1) d x+2\left(1+\frac{1}{3} \varepsilon^{3}\right) \int_{0}^{\infty} \hat{f}_{0}(x) \psi(x, 1) d x=\frac{1}{3} \varepsilon^{3}\left(\frac{1}{2}-r\right)+O\left(\varepsilon^{3}\right)$,

where algebraic manipulations leads the last equality. In accordance with the results on the truncated model, we found, that $r=1 / 2$ is critical, at $r<1 / 2$ the relative variance $R_{t}^{c}$ is positive, it increases for any $\varepsilon>0$, i.e. the population of identical particles is unstable, small perturbations disperse the mass distribution. At $r>1 / 2$ the relative variance $R_{t}^{c}<0$, which shows, that the population of identical particles is stable, the model is focusing.

\section{A6 Investigation of outliers in finite samples}

Let us have a sample with $N$ particles with $(N-1)$ having identical volume $X$. The last particle is an outlier with volume $a X$, where $a>>1$. In a single binary collision, a hit between particles with volume $X$ is called an A-type event, while a collision with the outlier being involved is a B-type event. Based on discrete probabilistic considerations, the probability of an A-type event equals $(N-2) / N$ and a B-type event is $2 / N$, respectively. In the A-type event the average size $\bar{X}$ of the particles with volume $X$ after the collision that lasts for $\Delta t$ reads

$\bar{X}=\frac{2(X-X / 2 \Delta t)+(N-3) X}{N-1}=X-\frac{X}{N-1} \Delta t$.

Computing $a X / \bar{X}$ and truncating the Taylor series expansion in $\Delta t$ after linear terms around the value $\Delta t=0$ yields the time

$a_{t}^{A}=\frac{a}{N-1}$. 
In case of the B-type event both the outlier and one of the small particles follow the compound kernel via:

$$
\begin{aligned}
(a X)_{t} & =-\frac{a^{1+r} X}{1+a}, \\
X_{t} & =-\frac{a^{1-r} X}{1+a} .
\end{aligned}
$$

The second equation is employed to compute the average volume of the small particles (i.e., $\bar{X}$ associated with this event).

5 Now we need to truncate the Taylor series of $\frac{a X-(a X)_{t} \Delta t}{X-\bar{X}}$ at $\Delta t=0$. After algebraic manipulations we find

$a_{t}^{B}=-\frac{a^{1+r}}{1+a}+\frac{a^{2-r}}{(1+a)(N-1)}$.

Considering the probabilities of events $\mathrm{A}$ and $\mathrm{B}$ we arrive to

$a_{t}=a \frac{N-2}{N-1}-2\left(\frac{a^{1+r}}{1+a}+\frac{a^{2-r}}{(1+a)(N-1)}\right)$.

Note that an increase in the value of $a$, i.e., $a_{t}>0$ implies that the outlier is getting further from the population. In the case of the $N \rightarrow \infty$ limit we find

$a_{t}=a\left(1-\frac{2 a^{r}}{1+a}\right)$.

Here the sign of the expression in the brackets determines the sign of $a_{t}$, which coincides with e.q. (8) in the text. One can also show, that if there exist $a^{c}>1$ such that $a_{t}=0$ at $a_{c}$, then $a_{t}>0$ for any $a>a^{c}$. Hence, we need the $a_{c}>1$ that makes the expression in the bracket vanish. Existence of such a critical value can be shown for the case with finitely many particles, too.

Author contributions. G.D. proposed the problem and supervised the research; A.Á.S. carried out the analytical and numerical study of the continuous model, T.J. developed the discrete numerical model; G.D., A.Á.S. and J.T. wrote the paper.

Competing interests. The authors declare that they have no competing interests.

Acknowledgements. The research reported in this paper was supported by the BME Water Sciences \& Disaster Prevention TKP2020 IE grant of NKFIH Hungary (BME IE-VIZ TKP2020) and the NKFIH grant K134199. 


\section{References}

Ashcroft, W.: Beach pebbles explained, Nature, 346, 227, 1990.

Bertoni, D., Sarti, G., Grottoli, E., Ciavola, P., Pozzebon, A., Domokos, G., and Novák-Szabó, T.: Impressive abrasion rates of marked pebbles on a coarse-clastic beach within a 13-month timespan, Marine Geology, 381, 175 - 180, 2016.

5 Bird, E.: Lateral Grading of Beach Sediments: A Commentary., Journal of Coastal Research, 12, 774-785, 1996.

Bloore, F. J.: The Shape of Pebbles, Math. Geol., 9, 113-122, 1977.

Bluck, B. J.: Sedimentation of beach gravels; examples from South Wales, Journal of Sedimentary Research, 37, $128-156,1967$.

Carr, A. P.: Size grading along a pebble beach; chesil beach, England, Journal of Sedimentary Research, 39, $297-311,1969$.

Cheng, Z. and Redner, S.: Scaling Theory of Fragmentation, Phys. Rev. Lett., 60, 2450-2453, 1988.

da Costa, F. P.: Mathematical Aspects of Coagulation-Fragmentation Equations, in: Mathematics of Energy and Climate Change, edited by Bourguignon, J.-P., Jeltsch, R., Pinto, A. A., and Viana, M., pp. 83-162, Springer International Publishing, Cham, 2015.

Dobkins, J. E. and Folk, R. L.: Shape development on Tahiti-Nui, Journal of Sedimentary Research, 40, 1167-1203, 1970.

Domokos, G. and Gibbons, G. W.: The evolution of pebble size and shape in space and time, Proc. Roy. Soc. A., 468, 3059-3079, 2012.

Domokos, G. and Gibbons, G. W.: Geometrical and physical models of abrasion, arXiv:1307.5633, 2013.

Driscoll, T. A., Hale, N., and Trefethen, L. N.: Chebfun Guide, Pafnuty Publications, Oxford, 2014.

Ernst, M. H. and Pagonabarraga, I.: The Nonlinear Fragmentation Equation, J. Physics A. Math. Theo., 40, F331-F337, 2007.

Firey, W. J.: Shapes of worn stones, Mathematika, 21, 1-11, 1974.

Gleason, R., Blackley, M. W. L., and Carr, A. P.: Beach stability and particle size distribution, Start Bay, Journal of the Geological Society, 131, 83-101, 1975.

20 Hansom, J. D. and Moore, M. P.: Size Grading along a Shingle Beach in Wicklow, Ireland, Journal of Earth Sciences, 4, 7-15, 1981.

Kuenen, P. H.: Experimental Abraison: 6. Surf Action, Sedimentology, 3, 29-43, 1964.

Kuenen, P. H. and Migliorini, C. I.: Turbidity Currents as a Cause of Graded Bedding, The Journal of Geology, 58, 91-127, 1950.

Landon, R. E.: An Analysis of Beach Pebble Abrasion and Transportation, The Journal of Geology, 38, 1930.

Lewis, W. V.: The Effect of Wave Incidence on the Configuration of a Shingle Beach, The Geographical Journal, 78, 129-143, 1931.

Lorang, M. and Komar, P.: Pebble shape, Nature, 347, 433-434, 1990.

Lubachevsky, B. D.: How to simulate billiards and similar systems, Journal of Computational Physics, 94, 255 - $283,1991$.

Meyer, C. J. and Deglon, D. A.: Particle collision modeling - A review, Minerals Engineering, 24, 719 - 730, 2011.

Neate, D. J. M.: Underwater pebble grading of Chesil Bank, Proceedings of the Geologists' Association, 78, 419 - 426, 1967.

Novák-Szabó, T., Sipos, A. Á., Shaw, S., Bertoni, D., Pozzebon, A., Grottoli, E., Sarti, G., Ciavola, P., Domokos, G., and Jerolmack, D. J.: Universal characteristics of particle shape evolution by bed-load chipping, Science Advances, 4, https://doi.org/10.1126/sciadv.aao4946, https://advances.sciencemag.org/content/4/3/eaao4946, 2018.

Orford, J. D.: Discrimination of particle zonation on a pebble beach, Sedimentology, 22, 441-463, 1975.

Sternberg, H.: Untersuchungen uber Langen-und Querprofilgeschiebefuhrender Flusse, Z. Bauwes., 25, 486-.506, 1875.

Szabó, T., Fityus, S., and Domokos, G.: Abrasion model of downstream changes in grain shape and size along the Williams River, Australia, Journal of Geophysical Research: Earth Surface, 118, 2059-2071, https://doi.org/10.1002/jgrf.20142, 2013.

Szabó, T., Domokos, G., Grotzinger, J. P., and Jerolmack, D. J.: Reconstructing the transport history of pebbles on Mars, Nature Communications, 6, 8366, 2015. 
https://doi.org/10.5194/esurf-2020-84

Preprint. Discussion started: 31 October 2020

(C) Author(s) 2020. CC BY 4.0 License.

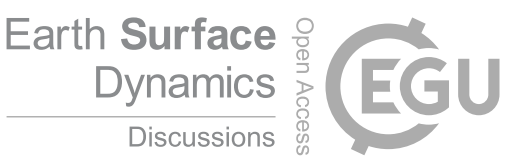

Uberoi, M. S.: Equipartition of energy and local isotropy in turbulent flows, Journal of Applied Physics, 28, 1165-1170, 1957.

Wald, Q. R.: The form of pebbles, Nature, 345, 211, 1990.

Williams, A. T. and Caldwell, N. E.: Particle size and shape in pebble-beach sedimentation, Marine Geology, 82, 199 - $215,1988$.

Yazawa, T.: More pebbles, Nature, 348, 398, 1990. 